\title{
L-H transition physics in hydrogen and deuterium: key role of the edge radial electric field and ion heat flux
}

\author{
F. Ryter, M. Cavedon, T. Happel, R.M. McDermott, E. Viezzer, \\ G.D. Conway, R. Fischer, B. Kurzan, T. Pütterich, G. Tardini, \\ M. Willensdorfer and the ASDEX Upgrade Team
}

Max-Planck-Institut für Plasmaphysik, D-85748 Garching, Germany

\begin{abstract}
.
Previous work carried out in the ASDEX Upgrade tokamak on the role of the edge radial electric field and ion heat flux in the $\mathrm{L}-\mathrm{H}$ transition physics in deuterium plasmas has been extended in hydrogen plasmas. Similar discharges were performed in the two gases providing a detailed comparison of the edge kinetic profiles and heat fluxes in L-mode up to the L-H transition, as the heating power is increased. At the L-H transition, the edge ion heat flux just inside the separatrix is about two times higher in hydrogen than in deuterium. However, the ion plasma parameters at the plasma edge, $T_{i}$ and $\nabla T_{i}$, as well as the radial electric field well, are found to be very similar in the two gases. The transport analysis based on this data reveals that, at the L-H transition, the ion heat transport at the plasma edge is about two times higher in hydrogen than in deuterium, in agreement with the well-known isotope effect of confinement and transport. This indicates that the higher power threshold in hydrogen is mainly due to the higher ion heat transport in this gas.
\end{abstract}

PACS: 52.55.-s 52.55.Dy 52.35.Ra 52.25.Fi

\section{Introduction}

The transition from the low to the high confinement mode in fusion plasmas, the L-H transition, occurs in general above a certain threshold in heating power, $P_{L-H}$. The density dependence of $P_{L-H}$ has been known for a long time to be non-monotonic, with a minimum at a density labelled $n_{e, \min }$. The power threshold increases on each side of this minimum, in the so-called low and high density branches. Older experimental results can be found in [1] and references therein, while the more recent ones are reported in $[2,3,4,5,6,7,8,9]$. The increase of $P_{L-H}$ in the low density branch is observed in most of the tokamaks but with 
variable strength, whereby the effect is particularly strong in plasmas with electron heating, e.g. electron cyclotron resonant heating $(\mathrm{ECRH}),[2,5,6]$. A former suggestion that this could be due to the reduction of the electron-ion coupling at low density, [10], has indeed been confirmed by recent results in ASDEX Upgrade which indicate that the edge ion heat flux plays a key role, [11].

The power threshold also exhibits a universal $1 / m_{i}$ ion mass dependence, known for almost three decades, $[12,13,14,15,8]$. Thus, $P_{L-H}$ is about two times higher in hydrogen than in deuterium. Note that the $1 / m_{i}$ dependence also applies to tritium, [14], but not to helium, $[16,5,17]$

At the L-H transition an edge transport barrier for heat and particles, caused by the reduction of turbulence-driven transport, is formed just inside the last closed magnetic surface. Presently, the most favoured paradigm to explain this phenomenon is based on turbulence reduction induced by radially sheared $E \times B$ flow, [18]. In the narrow region at the very edge of the plasma, where the L-H transition occurs, the $E_{r}$ profile exhibits a clear well such that $\nabla E_{r}$ induces a perpendicular sheared flow which stabilises the turbulence. This hypothesis has been confirmed experimentally for spontaneous L-H transitions, originally in DIII-D, [19], later in most of the devices, see e.g. reviews [20,21]. The $E_{r}$ well is weakly pronounced in L-mode, much deeper in H-modes and in-between just before the L-H transition, [21, 22, 23]. Neoclassical theory predicts that the edge $E_{r}$ is essentially induced by the main ions, here deuterium or hydrogen, [24], such that it is mainly determined by the gradient of the main ion pressure, $E_{r} \approx \nabla p_{i} /\left(e \cdot n_{i}\right)$, where $n_{i}$ is the ion density and $e$ the elementary charge, [25, 26, 23] and references therein.

In general the transition to $\mathrm{H}$-mode is achieved by increasing the heating power, whereby the plasma density almost does not change before the L-H transition, but $T_{i}$ and $\left|\nabla T_{i}\right|$ are expected to increase. The $E_{r}$ well, which becomes deeper, eventually induces the transition to $\mathrm{H}$-mode. Therefore one expects that the ion heat flux at the plasma edge, $Q_{i, e d g e}$, strongly contributes to the L-H physics mechanism, as documented in our previous work, [11], in which we provided experimental evidence for the key role played by this quantity in the L$\mathrm{H}$ transition. This explains the increase of $P_{L-H}$ in the low density branch by the fact that the electron-ion collisional coupling is reduced and that more heating power is required to establish the necessary edge ion heat flux. We found recently in ASDEX Upgrade that both $P_{L-H}$ and $n_{e, \min }$ decrease as plasma current is reduced, [8]. This behaviour is also explained 
by the edge ion heat flux through the energy confinement dependence on plasma current, [11]. It should be underlined that the increase of the heating power on the path to the L-H transition also enhances the turbulence level which excites zonal flows and geodesic acoustic modes leading to self-induced transient reduction of turbulence and transport through the predator-prey mechanism, indeed observed in several fusion devices before the L-H transition, $[27,28,29,30,31,32,33]$. This phenomenon is reflected in phases preceding the L$\mathrm{H}$ transition during which limits cycle oscillations arising from the interplay between $E_{r}$ turbulence and transport reduction are observed, also named IM-mode, [28], or I-phase, [30]. The turbulence-induced flows, which are superimposed to the neoclassical one, have been proposed as a possible triggering mechanism of the L-H transition, [34], whereby the neoclassical flow driven by the ion pressure gradient remains the only player after the $\mathrm{L}-\mathrm{H}$ transition as turbulence has been strongly reduced and the corresponding flows disappeared. In in section 3 of the present article, we extend our previous work with analyses of the electron-ion collisional energy exchange in the new hydrogen discharges and an investigation of $E_{r}$ in the L-mode as the heating power is increased up to the L-H transition. In section 4, we complete the previous results on the ion heat flux at the L-H transition carried out in deuterium with the data obtained in hydrogen. The experimental set-up and conditions are described in the forthcoming section.

\section{Experimental setup and analysis methods}

The experiments have been performed in ASDEX Upgrade, a divertor tokamak with major and minor radii $\mathrm{R}=1.65 \mathrm{~m}$ and $\mathrm{a}=0.5 \mathrm{~m}$ respectively. The data presented here are taken from deuterium and hydrogen plasmas in the standard lower single null magnetic configuration with the ion $\nabla B$ drift directed towards the active $\mathrm{X}$-point providing the usual low L-H transition power threshold for the two isotopes respectively. The deuterium data presented in reference [11] are extended in the present work by equivalent analyses in hydrogen, yielding a direct comparison between the two ion species. In the discharges considered here, the purity of the dominant species, D or H, was about $90 \%$, as deduced from the low energy neutral fluxes measured by the neutral particle analysers.

The actual thermal electron temperature, $T_{e}$, is deduced from the radiation temperature measured by the electron cyclotron emission (ECE) diagnostic using a forward modelling which also account for the the shine-through effect at the plasma edge and provides also 
there reliable $T_{e}$ values, [35]. It is implemented in the integrated data analysis framework (IDA) which also yields the density profile combining the interferometry data with those from the plasma edge yielded by the Lithium beam and/or edge Thomson scattering, [36]. The ion temperature, $T_{i}$, and the plasma rotation components, $\mathrm{v}_{\text {tor }}$ and $\mathrm{v}_{\text {pol }}$, are provided by the charge exchange recombination spectroscopy (CXRS) core and edge diagnostics with a time resolution of $4 \mathrm{~ms}$ in the core and $2 \mathrm{~ms}$ in the edge, [37], the latter reduced down to $100 \mu \mathrm{s}$ since the 2014 campaign, [38]. This enables us since 2011 to measure these quantities reliably in ECRH heated plasmas using short NBI blips, [39]. The radial electric field at the plasma edge can be deduced from the CXRS data applying the ion force balance, but it can also be derived from the Doppler reflectometry measurement, assuming that the phase velocity of the turbulence is much smaller than the back-ground flow, [40].

The threshold power, $P_{L-H}$, is obtained, as usual, from $P_{l o s s}=P_{\text {heat }}-d W / d t$ at the $\mathrm{L}-\mathrm{H}$ transition, where $P_{\text {heat }}$ is the sum of all the heating powers in which possible losses and absorption coefficients are taken into account, $\mathrm{W}$ is the plasma energy. As will be shown below, important quantities are the surface-integrated ion and electron heat fluxes at the plasma edge, $Q_{i, e d g e}$ and $Q_{e, e d g e}$, which are taken at $\rho_{\text {pol }} \approx 0.98$, where $\rho_{\text {pol }}$ is the usual normalised poloidal flux radius. These fluxes are provided by power balance analyses using the TRANSP code, [41]. As discussed in detail in [11], under the experimental conditions used here, an essential term in the power balance is the collisional electron-ion energy exchange, $p_{e i} \propto n_{e} n_{i}\left(Z_{i}^{2} / m_{i}\right)\left(T_{e}-T_{i}\right) T_{e}^{-3 / 2}$, which increases with $\left(T_{e}-T_{i}\right)$, but exhibits a saturation and rollover at high $T_{e}$ due to $T_{e}^{-3 / 2}$. Note that due to its $1 / m_{i}$ dependence $p_{e i}$ is two times higher in hydrogen than in deuterium. As discussed in [11], here also the contribution of $p_{e i}$ to the heat fluxes can only be calculated with sufficient accuracy only if $\left(T_{e}-T_{i}\right)$ is larger than the experimental uncertainties on the temperatures. This limits the analysis to densities below roughly $4 \times 10^{19} \mathrm{~m}^{-3}$, therefore mainly in the low density branch of $P_{L-H}$. For our studies in the low density branch, the preferred heating method is ECRH as the density can be better controlled than with NBI and because $p_{e i}$ can be calculated with good accuracy thanks to the fact that $\left(T_{e}-T_{i}\right)$ is generally larger than the experimental uncertainties over a large part of the radius. In the experiments described here, both in hydrogen and deuterium, the ECRH power has been injected using the standard second harmonic scheme which provides pure electron heating with $100 \%$ absorption and a narrow deposition profile [42]. The power was deposited in the central part of the plasma $\left(\rho_{p o l}<0.35\right)$, for all the cases presented here. 


\section{Relation between ion heat flux and $E_{r}$ at the plasma edge in hydrogen}

\subsection{Behaviour of $p_{e i}$ and $Q_{i}$}

A hydrogen discharge in the low density branch, run at $1 \mathrm{MA}$ and $\left|B_{T}\right|=2.5 \mathrm{~T}$, is illustrated in figure 1 by a few time traces.

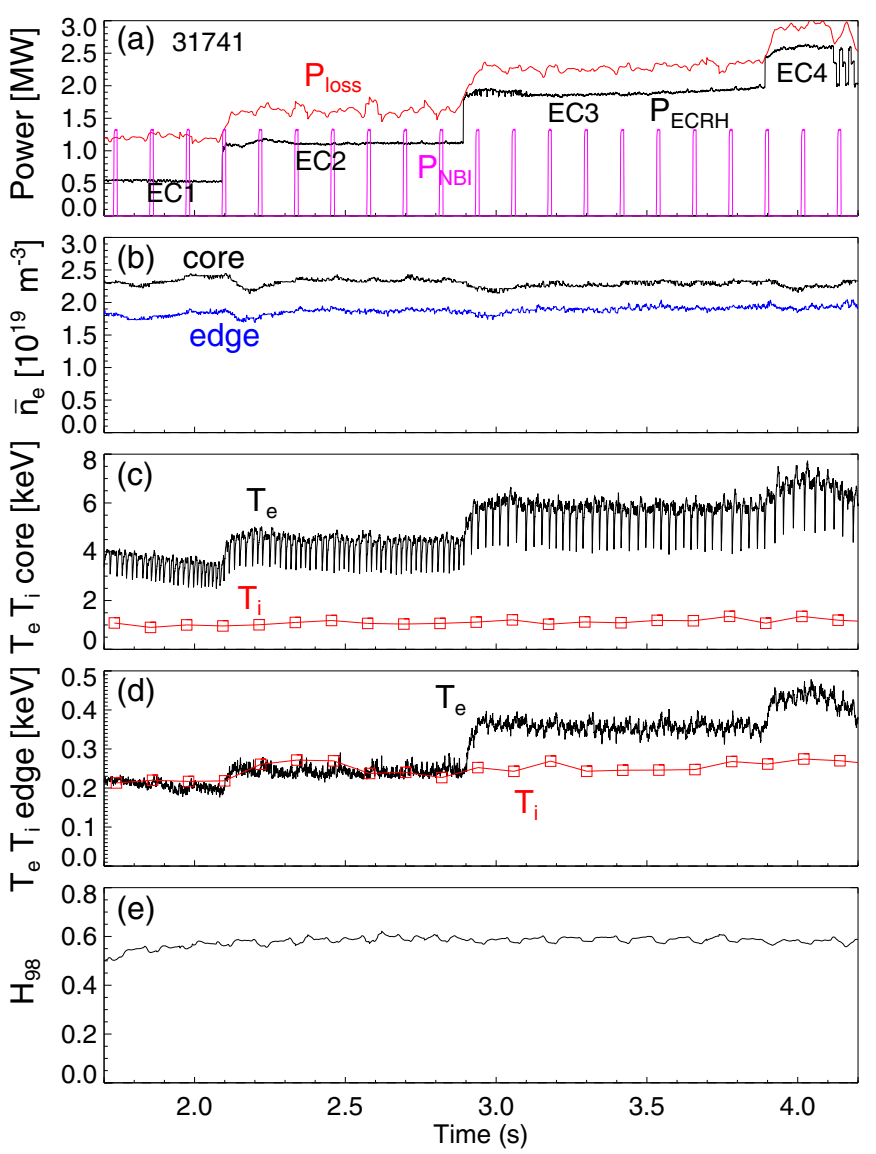

Figure 1. Time traces of a discharge in hydrogen with steps in $P_{E C R H}$. Panel a: heating powers; Panel b: line-averaged densities of two channels (see text for details); Panel c: central electron and ion temperatures; Panel d: edge electron and ion temperatures $\left(\rho_{\text {pol }} \approx 0.98\right)$; Panel e: $H$-mode enhancement factor $H_{98 y 2}$.

Panel (a) displays the heating powers. The auxiliary ECRH power has been increased in four steps, labelled EC1 to EC4 with respective powers of $0.5,1.1,1.9$ and $2.5 \mathrm{MW}$. The last step is very close to the L-H transition as indicated by the appearance of short I-phases, but, limited in time by ECRH trips, does not yield sufficiently good edge data and can only be partly used in the following analyses. The NBI blips enable the CXRS measurements. The loss power increases following the steps of $P_{E C R H}: 1.2,1.6,2.3$ and $2.9 \mathrm{MW}$. In panel $\mathrm{b}$ of 
figure 1 we show the line-averaged density from two interferometer channels, labelled core and edge, with tangency radii $\rho_{\text {pol }} \approx 0.1$ and $\rho_{\text {pol }} \approx 0.87$ respectively. The diagnostic details can be found in [43]. The core line-averaged density in this discharge is $2.4 \times 10^{19} \mathrm{~m}^{-3}$, which is clearly in the low density branch, see [8] or figure 8 below. The density was kept constant with gas puffing by feedback on $\bar{n}_{e, c o r e}$ such that it tends to decrease at each $P_{E C R H}$ step but is brought back to the requested value by the control system. Note that the ratio $\bar{n}_{e, \text { core }} / \bar{n}_{e, e d g e}$ is slightly reduced in each of the ECRH power steps indicating a weak flattening of the density profile with increasing ECRH power, in agreement with previous studies in L-mode, [44, 45]. Panel (c) displays the central electron and ion temperatures. As expected for ECRH heating, $T_{e}$ increases significantly with $P_{E C R H}$, while the changes in $T_{i}$ are very small, also shown by the profiles in figure 2. The modulation of the electron temperature is caused by the sawteeth. The edge temperatures, taken at about $\rho_{\text {pol }}=0.98$ are shown in panel (d). At low ECRH power the two temperatures are about equal, but $T_{e, e d g e}$ increases much more than $T_{i, e d g e}$ as $P_{E C R H}$ is increased and is clearly higher during the last two ECRH steps. This large difference between $T_{e}$ and $T_{i}$ at the plasma edge under such conditions has already been pointed out for deuterium discharges in [22]. Finally, panel (e) indicates that, with $H_{98 y 2} \approx 0.6$, whereby the scaling takes the isotope confinement dependence into account, the global confinement time is in the usual L-mode range.

The data have been analysed in the four $P_{E C R H}$ levels and the temperature profiles are plotted in figure 2. Clearly $T_{e}$ is larger than $T_{i}$ and this difference increases with increasing $P_{E C R H}$, while $T_{i}$ varies very little, which is a property also observed in similar discharges in deuterium. The ECRH power deposition at $\rho_{\text {pol }} \approx 0.08$ is schematically indicated in the figure. Note that for EC2, EC3 and EC4 $T_{e}$ is higher than $T_{i}$ up to the plasma edge, providing good conditions to calculate $p_{e i}$ and $Q_{i, e d g e}$.

The profiles of $p_{e i}$, the local power density of the collisional electron-ion energy transfer, and the corresponding surface-integrated ion heat flux, $Q_{i}$, are plotted in figure $3 \mathrm{a}$ and $\mathrm{b}$ respectively for the four ECRH steps. In the plasma core, $T_{e}$ is already high during EC1 and due to the $T_{e}^{-3 / 2}$ dependence, $p_{e i}$ decreases as $P_{E C R H}$ and $T_{e}$ increase: increasing $P_{E C R H}$ leads to a decrease of $Q_{i}$ in the central plasma. Further out $\left(\rho_{p o l}>0.5\right), T_{e}-T_{i}$ dominates the dependence of $p_{e i}$ which increases with ECRH power and this leads to higher $Q_{i, e d g e}$ with more ECRH power. However, for the last ECRH step the decrease in the central plasma cannot be completely compensated by the outer part and $Q_{i, e d g e}$ is not higher than in step EC3. It is 


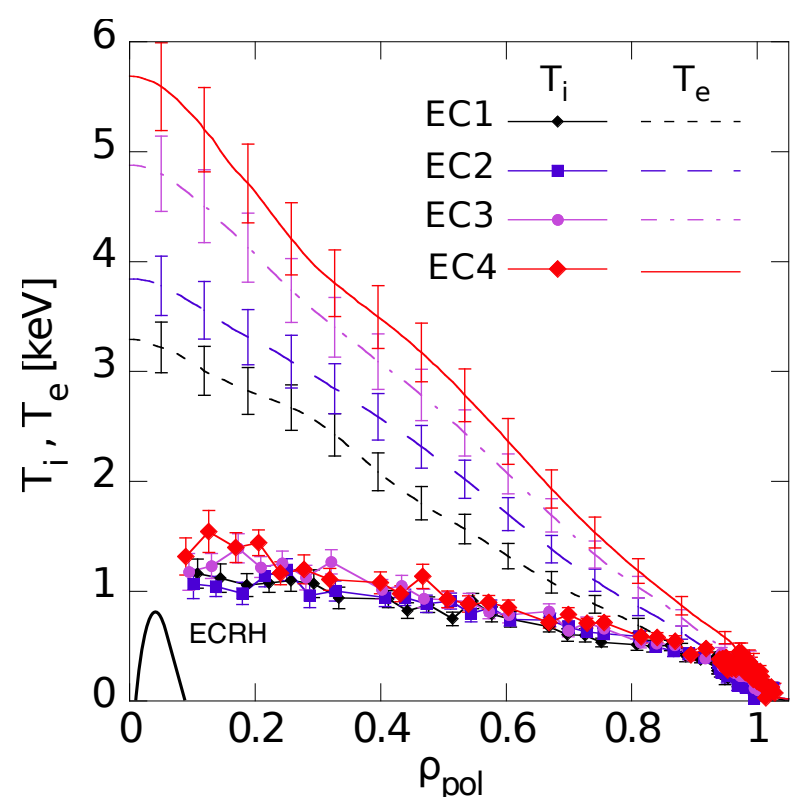

Figure 2. $T_{e}$ and $T_{i}$ profiles for the four $P_{E C R H}$ values of the hydrogen discharge 31741 from figure 1. The $T_{e}$ profiles are the IDA fits.

worth underlying that in this discharge $Q_{i, e d g e}$ increases by about $80 \%$, from 0.52 to $0.95 \mathrm{MW}$, while $P_{\text {loss }}$, which is pure electron heating $\left(P_{O H}+P_{E C R H}\right)$, is multiplied by almost a factor of three, from 1.2 to $2.9 \mathrm{MW}$. The behaviour of $p_{e i}$ explains the rather weak increase of the ion heat flux with $P_{E C R H}$. The decrease of $p_{e i}$ in the central plasma with increasing $P_{E C R H}$ and $T_{e}$ is in agreement with the fact that $T_{i}$ in the core varies very little as $P_{E C R H}$ is increased. This weak dependence of $Q_{i, e d g e}$ on ECRH power at low density explains that long I-phases are mainly found in the low density branch and over a wide window of heating power, [30].

At higher density the situation is different as illustrated in figures 4 and 5 for a discharges at $\bar{n}_{e} \approx 3.95 \times 10^{19} \mathrm{~m}^{-3}$. This discharge was heated with three ECRH steps and the L$\mathrm{H}$ transition occurred during the last step. The values of $P_{E C R H}$ were $0.7,1.4$ and 2.1 MW, yielding for $P_{\text {loss }} 1.70,1.95$ and $2.65 \mathrm{MW}$ respectively, whereas the last value of $P_{\text {loss }}$ corresponds to $P_{L-H}$. As indicated by figure 4 the electron temperature is of course overall about two times lower than at lower density and closer to $T_{i}$ which does not change much with density. The ECRH power is deposited at $\rho_{\text {pol }} \approx 0.3$ which explains the flat $T_{e}$ profiles in the central plasma. Panel a of figure 5 shows that, in contrast to the low density case, $p_{e i}$ increases with $P_{E C R H}$, even in the center, because it is below the saturation induced by $T_{e}^{-3 / 2}$ over the whole radius. Panel b indicates that $Q_{i}$ increases significantly over the whole radius as $P_{E C R H}$ is increased and thus, even for pure electron heating, $Q_{i, e d g e}$ is more strongly linked 

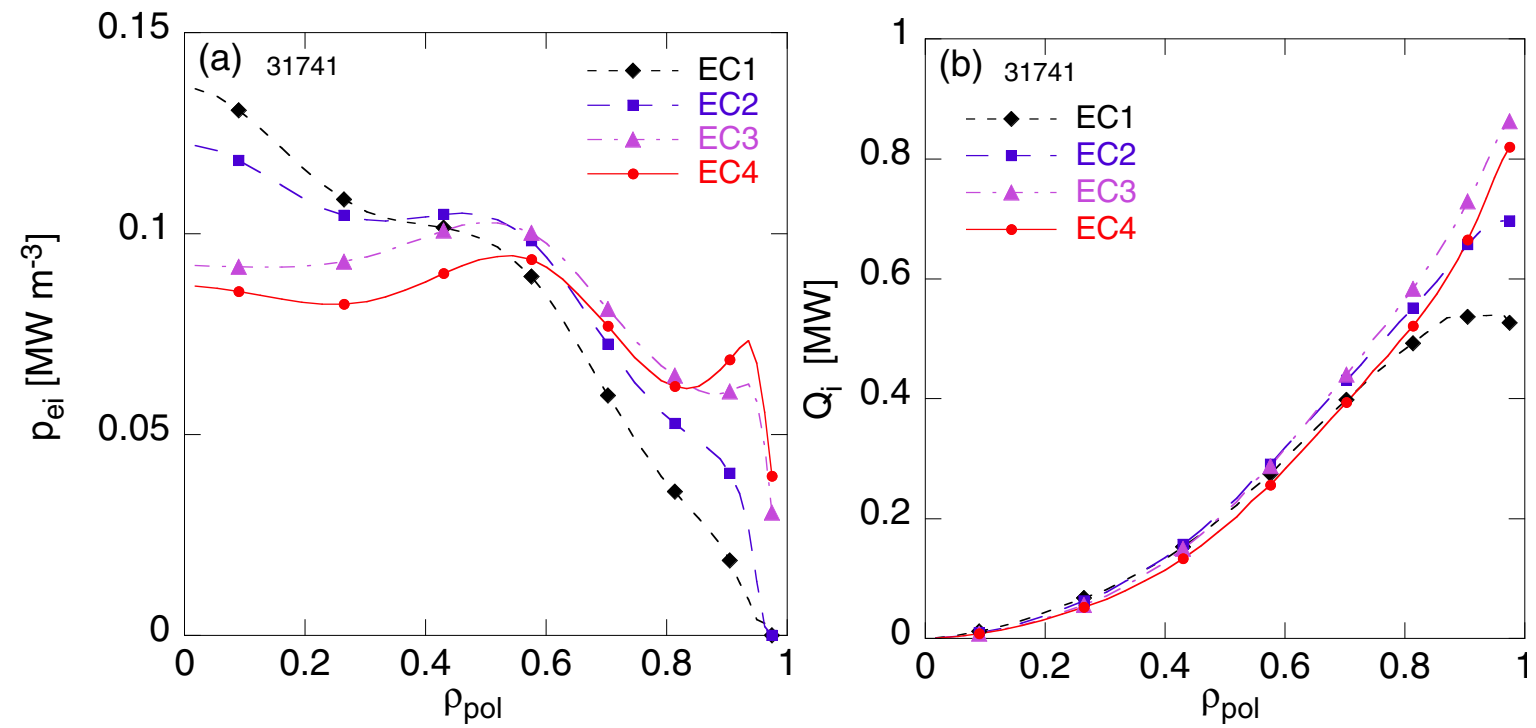

Figure 3. Panel a: $p_{e i}$ profiles for the four ECRH steps of the hydrogen discharge 31741, corresponding to the temperature profiles from figure 2. Panel b: Corresponding profiles of the surface-integrated ion heat flux. The symbols help identifying the different cases and do not represent measurement points.

to the heating power than at low density.

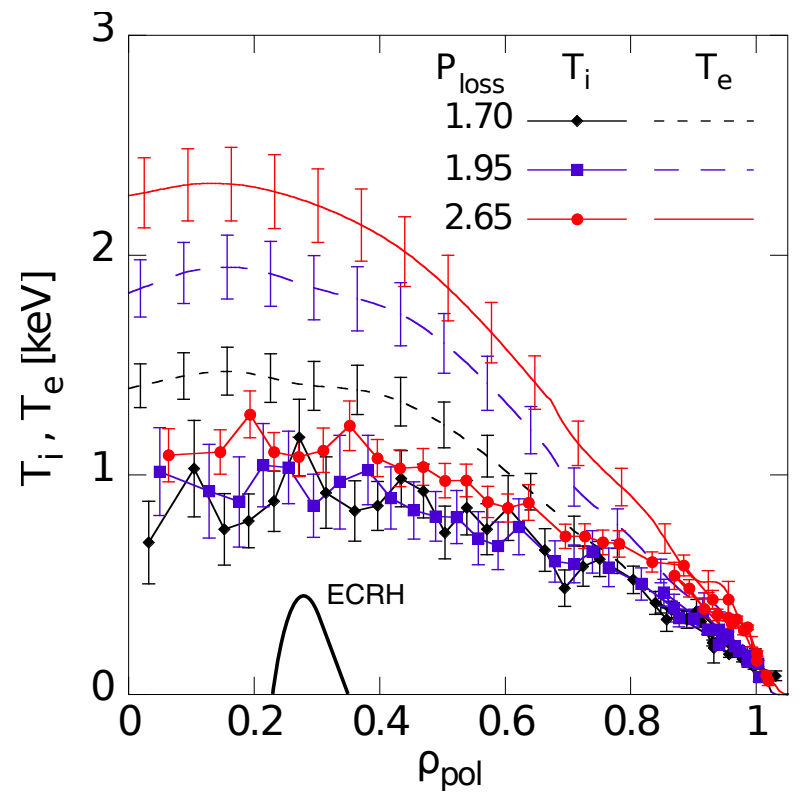

Figure 4. $T_{e}$ and $T_{i}$ profiles for the three $P_{E C R H}$ values of the hydrogen discharge 27368. The $T_{e}$ profiles are the IDA fits. 

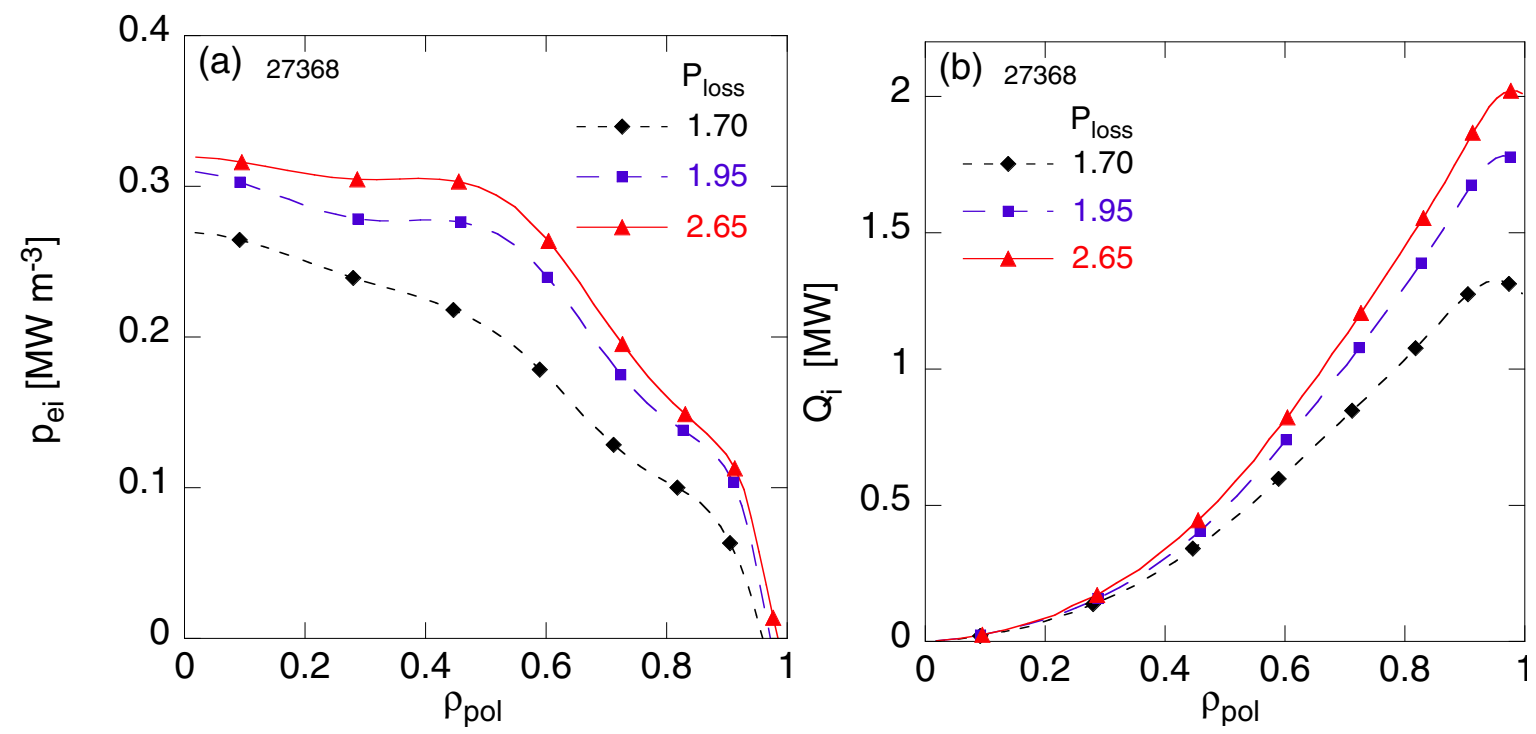

Figure 5. Panel a: $p_{e i}$ profiles for the three ECRH steps of the hydrogen discharge 27368, corresponding to the temperature profiles from figure 4. Panel b: Corresponding profiles of the surface-integrated ion heat flux. The symbols help identifying the different cases and do not represent measurement points.

\subsection{Radial electric field}

Under the assumption that the radial electric field well plays an important role in the stabilisation of turbulence and in the transition to $\mathrm{H}$-mode, it is instructive to analyse the evolution of $E_{r}, T_{i}$ and $\nabla T_{i}$ at the plasma edge in an L-mode $P_{E C R H}$ scan, here discharge 31741 already described above. The $E_{r}$ profiles yielded by Doppler reflectometry, $E_{r}^{D R}$, for the first and third $P_{E C R H}$ steps (EC1 and EC3) are shown in figure $6 \mathrm{a}$ and $\mathrm{b}$, together with the neoclassical values, $E_{r}^{N E O}$, calculated with NEOART, [46], based on the experimental data. The same density profile has been used for the two analyses. The calculation of $E_{r}^{N E O}$ includes all the terms, including the toroidal rotation which has been measured to be around zero with uncertainties of about $\pm 5 \mathrm{~km} / \mathrm{s}$ in the radial region for $\rho_{\text {pol }}>0.94$. These are reflected by the error bars in the plots. We verified that the uncertainties on the effective charge have a small impact on the $E_{r}^{N E O}$ profile. The error bars are quite large for $E_{r}^{N E O}$ in particular because the the signal-to-noise ratio of the CXRS data is not very good when short beam blips are used. The outer part $\left(\rho_{\text {pol }}>0.995\right)$ of the $E_{r}^{N E O}$ profiles is cut because the calculation becomes too uncertain due to the poorly defined $\nabla T_{i}$, underlined by the increase of the error bars. In addition, further outwards, the boundary condition of $E_{r}$ might be influenced by the scrape- 
off layer. Within the errors the profiles of $E_{r}^{D R}$ and $E_{r}^{N E O}$ are in relatively good agreement for panel a, but clearly poorer for panel b, whereas the flattening of $E_{r}^{N E O}$ can be partly attributed to the somewhat higher edge density mentioned above.
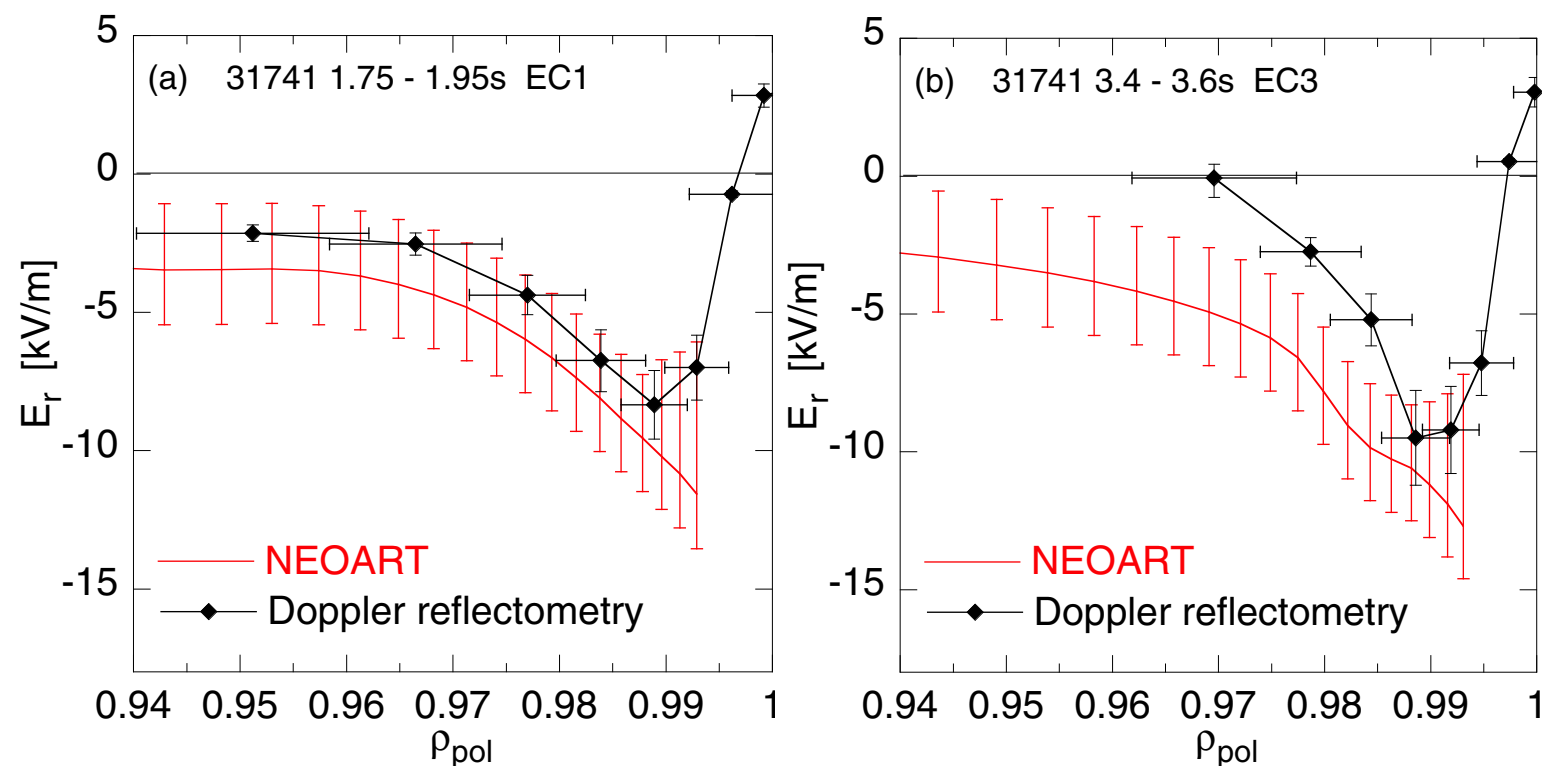

Figure 6. $E_{r}$ profiles from NEOART and Doppler reflectometry for the EC1 and EC3 phases of the hydrogen discharge 31741.

The $E_{r}^{D R}$ measurements suggest a somewhat narrower well with more heating power. This could be an important feature as $\nabla E_{r}$ is considered as the key element in the turbulence stabilisation which leads to the L-H transition, but further studies will be necessary to assess this point. Nevertheless, the minimum of the $E_{r}^{D R}$ profile is rather close to the neoclassical value in the two cases. This is in agreement with the other results in ASDEX Upgrade: indeed, in all the studies of $E_{r}$ performed in L-mode and H-mode in this tokamak, the deviations of the measured $E_{r}$ profile from $E_{r}^{N E O}$ are small, [23, 47]. For the L-H transition specifically, previous comparisons suggested that the $E_{r}$ well at the $\mathrm{L}-\mathrm{H}$ transition was close to the neoclassical prediction, [22], and the recent results using the new fast (100 $\mu$ s) edge CXRS diagnostic clearly indicate that during the I-phase and around the L-H transition the measured $E_{r}$ profile and $E_{r}^{N E O}$ are close to each other within the error bars. The reader if referred to [38] for an extensive description and discussion of these results. Coming back to figure 6: despite the uncertainties and although $E_{r}^{N E O}$ exhibits a somewhat more negative value at the minimum of $E_{r}^{D R}$, both profiles indicate that the $E_{r}$ well minimum is only weakly deeper at higher power. This can be investigated with our experimental data as follows. 
According to neoclassical theory, the main contribution to the edge $E_{r}$ arises from the diamagnetic term, $\nabla p_{i} /\left(e \cdot n_{i}\right)=\left(\nabla T_{i}+T_{i} \nabla n_{i} / n_{i}\right) / e,[26]$. It is therefore instructive to investigate the experimental values of $T_{i, e d g e}$ and $\nabla T_{i, e d g e}$ taken close to the position of the $E_{r}$ minimum, at $\rho_{\text {pol }} \approx 0.98$. They are plotted versus $Q_{i, e d g e}$, taken at the same radial position, in figure $7 \mathrm{a}$ and $\mathrm{b}$ respectively, for the above discharge and a similar one, yielding a bit more statistics. Both quantities increase by about $30 \%$ for an increase in $Q_{i, e d g e}$ of about $80 \%$. This is a rather weak increase which partly explains the weak deepening of the $E_{r}$ profile as power is increased.
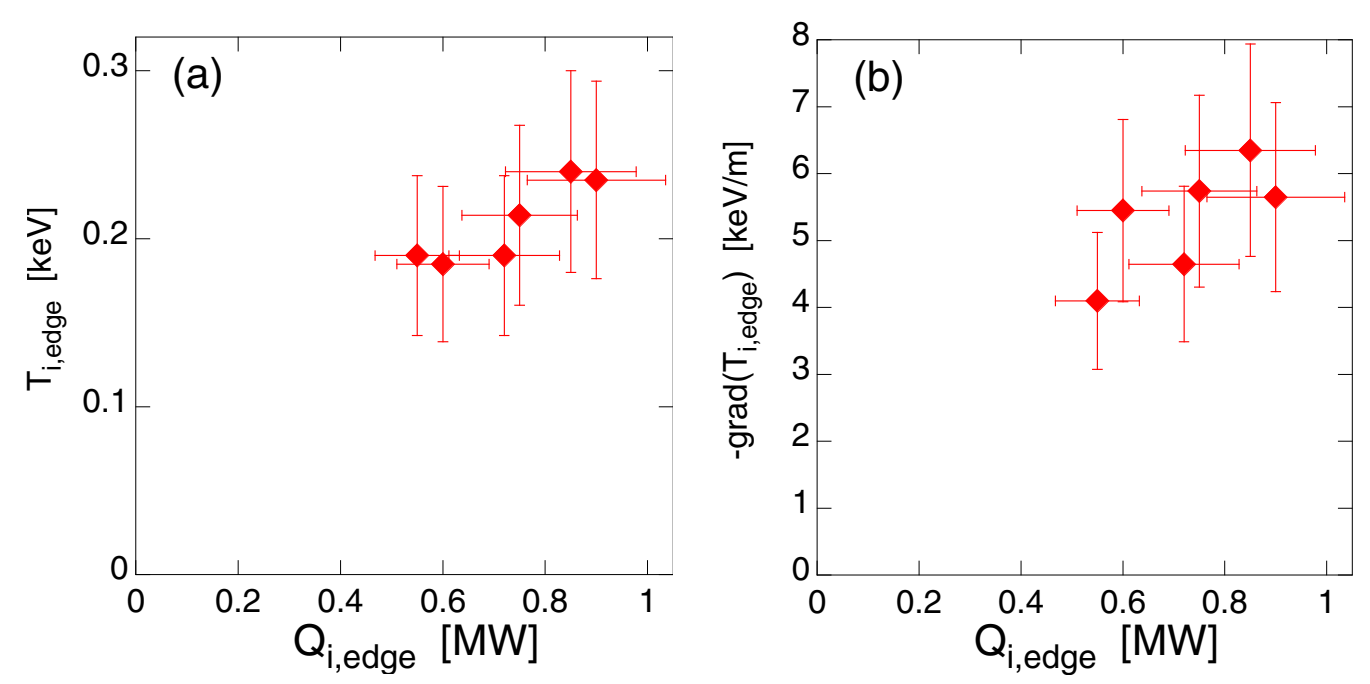

Figure 7. Panels $a$ and $b$ show respectively $T_{i, \text { edge }}$ and $-\nabla T_{i, \text { edge }}$ versus $Q_{i, \text { edge }}$ in L-mode $P_{E C R H}$ scans at $n_{e} \approx 2.4 \times 10^{19} \mathrm{~m}^{-3}$ of the hydrogen discharges 31739 and 31741. All the data are taken at $\rho_{\text {pol }} \approx 0.98$.

\section{Power threshold and edge ion heat flux: hydrogen and deuterium comparison}

We have shown in reference [11] that, in deuterium plasmas, the edge ion heat flux at the L$\mathrm{H}$ transition, $Q_{i, e d g e}^{L-H}$ (taken at $\rho_{\text {pol }} \approx 0.98$ ), increases linearly with the line-averaged density: $Q_{i, e d g e}^{L-H, f i t}=0.18 \bar{n}_{e}$, in MW and $10^{19} \mathrm{~m}^{-3}$. Hydrogen discharges similar to those described in the previous section at various densities yield data which can be compared to the results obtained in deuterium. This is illustrated in figure 8 where $P_{L-H}$ and $Q_{i, e d g e}^{L-H}$ from the recent hydrogen study are plotted together with the deuterium data at $1 \mathrm{MA}$ from [11].

As is well-known and reported earlier for ASDEX Upgrade in [5, 8], $P_{L-H}$ in hydrogen is higher than in deuterium by about a factor of two and also exhibits a minimum at $n_{e, \min }$, 


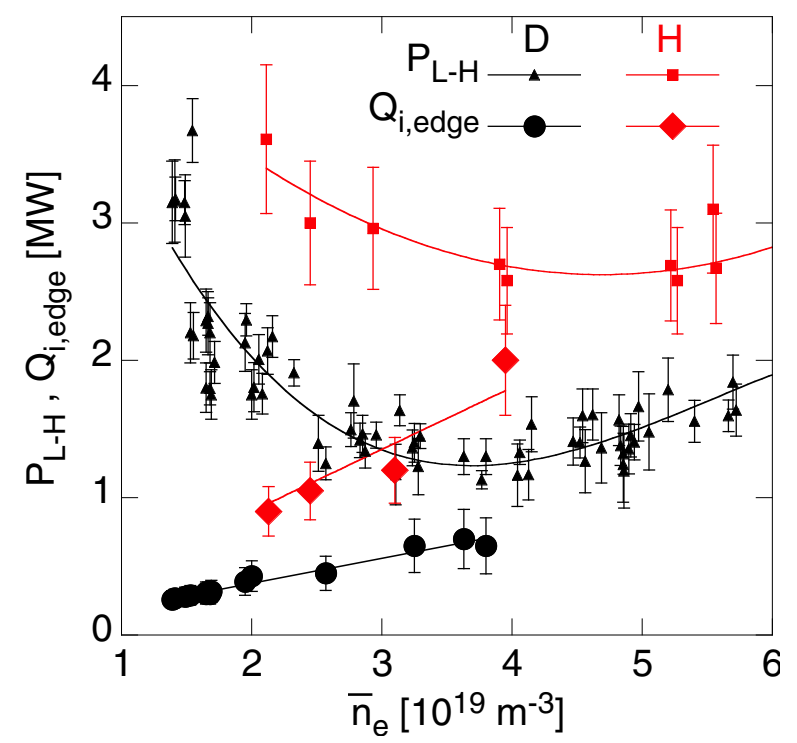

Figure 8. $P_{L-H}$ and $Q_{i, e d g e}\left(a t \rho_{p o l} \approx 0.98\right)$ at the $L-H$ transition versus line-averaged density in hydrogen and deuterium. All discharges at $1 M A,\left|B_{T}\right| \approx 2.5$ T. Deuterium data from [11].

whose value is somewhat higher than that for deuterium, a trend also observed in JET, [48]. As explained in [11] we interpreted the existence of $n_{e, \min }$ as due to the effect of the electronion coupling, whereby $n_{e, \min }$ corresponds to a given value of the ratio $\tau_{E} / \tau_{e i}$. Here $\tau_{E}$ is the energy confinement time and $\tau_{e i}$ the volume-averaged electron-ion energy exchange time. Based on the confinement and power threshold scalings, a formula for $n_{e, \min }$ could be derived. Including the isotope effects in the derivation reveals that the linear dependences of $\tau_{e i}$ and $P_{L-H}$ on the ion mass cancel each other almost exactly. The mass dependence of confinement remains and yields for $n_{e, \min }$ a weak mass dependence of the order of $M^{-0.05}$ which indeed yields a somewhat higher value in hydrogen. This is, therefore, an indirect but convincing confirmation of the hypothesis that $n_{e, \min }$ is related to the electron-ion collisional energy exchange.

An important aspect of our study deals with the comparison of the edge ion heat flux at the $\mathrm{L}-\mathrm{H}$ transition in the two gases. Figure 8 indicates that, as in deuterium, the edge ion heat flux in hydrogen at the L-H transition increases roughly linearly with density. In agreement with the high values of $P_{L-H}, Q_{i, e d g e}^{L-H}$ is about a factor of two higher in hydrogen than in deuterium and the fit forced through the origin reads $Q_{i, e d g e}^{L-H, f i t}=0.45 \bar{n}_{e}$, in MW and $10^{19} \mathrm{~m}^{-3}$. Therefore, in deuterium and hydrogen, both $P_{L-H}$ and $Q_{i, e d g e}^{L-H}$ exhibit respectively the same qualitative behaviour in the low density branch. It should be underlined that the extrapolation of the 
$Q_{i, e d g e}^{L-H, f i t}$ formulae to higher density for the two gases remains below the respective values of $P_{L-H}$, indicating that there are not unrealistic.

The edge electron heat flux at the L-H transition for the same data, $Q_{e, e d g e}^{L-H}$ at $\rho_{\text {pol }} \approx 0.98$, has also been analysed, as already reported for deuterium in [11]. It was shown therein that $Q_{e, e d g e}^{L-H}$ does not yield a coherent pattern, in particular when comparing the data for discharges at 0.6 and 1.0 MA, or ECRH and NBI, indicating that the electron channel most probably does not play an important role in the L-H transition mechanism. In the present work we compare $Q_{e, e d g e}^{L-H}$ in hydrogen and deuterium, as illustrated in figure 9 which displays $Q_{e, e d g e}^{L-H}$ versus density, for the corresponding $Q_{i, e d g e}^{L-H}$ points of figure 8 . For clarity, we present only the fits of $P_{L-H}$ and not the data points which are already plotted in figure 8 . In the two gases $Q_{e, e d g e}^{L-H}$ decreases as density is increased which is opposite to the dependence of the ion channel. The decrease of $Q_{e, e d g e}^{L-H}$ with density is very similar in the two gases and, despite the higher values of $P_{L-H}$ in hydrogen, the $Q_{e, e d g e}^{L-H}$ values are marginally higher than in deuterium which is due to the stronger electron-ion coupling. It should be pointed out that with ECRH, $Q_{e, e d g e}$ always decreases and $Q_{i, e d g e}$ always increases when the density is increased. The experiments reported in [11] in which the L-H transition was induced by increasing the density at fixed heating power demonstrated that it is achieved by increasing the edge ion heat flux while the electron heat flux was correspondingly reduced. Therefore, the similarity of $Q_{e, e d g e}^{L-H}$ in the two gases should not be interpreted as an indication for an important contribution of this quantity in the L-H transition physics. Finally, it is noted that the values of $Q_{e, e d g e}^{L-H}$ are very low at $\bar{n}_{e} \approx 4 \times 10^{19} \mathrm{~m}^{-3}$, the error bars then very large there, which is due to those on $p_{e i}$ and in addition to to those on the radiation losses. This indicates that these conditions are clearly at the upper boundary of the density for the analysis of the edge electron heat flux and the separation between the heat channels.

As mentioned above, all the results discussed here, in both hydrogen and deuterium, were obtained from discharges using ECRH deposited in the central part of the plasma. One might speculate that off-axis ECRH deposition could extend the field of investigations. A limited number of experiments carried out in deuterium during the 2009 campaign with offaxis ECRH at $\rho_{\text {pol }} \approx 0.7$ exhibited only small changes in $P_{L-H}$. This is mainly due to the fact that, with off-axis ECRH, the $T_{e}$ profile flattens such that the reduction of $p_{e i}$ in the central plasma is compensated by an increase in the outer part and $Q_{i, e d g e}$ varies little. At low density, changes of the density profile correlated with the $T_{e}$ profile also contribute. Overall, 


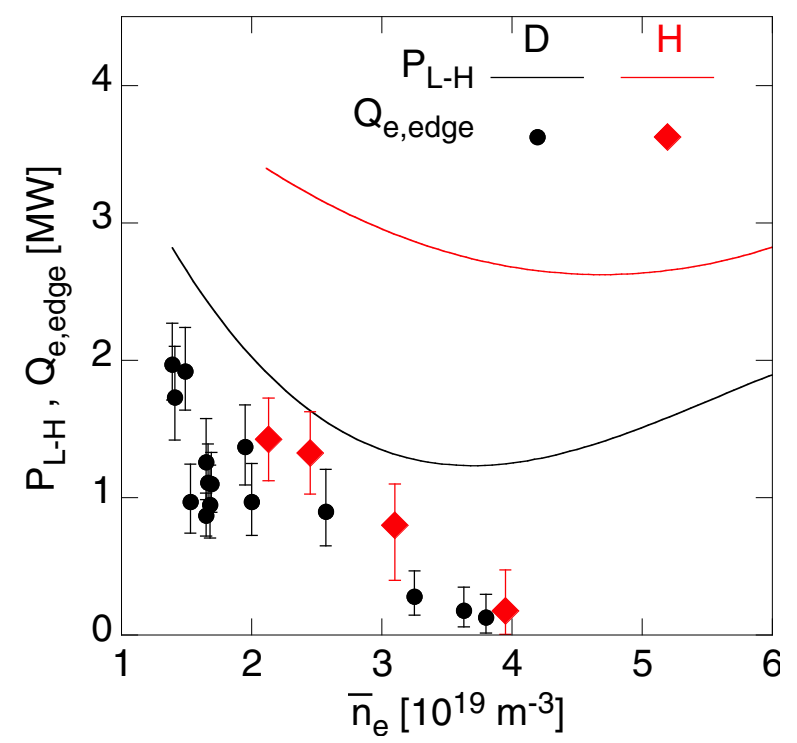

Figure 9. Fits of $P_{L-H}$ and $Q_{e, \text { edge }}\left(a t \rho_{p o l} \approx 0.98\right)$ at the $L-H$ transition versus lineaveraged density in hydrogen and deuterium. All discharges at $1 M A,\left|B_{T}\right| \approx 2.5 T$, corresponding to those of figure 8. Deuterium data from [11].

the comparison between on-axis and off-axis deposition does not seem to be a way to further assess the role of $Q_{i, e d g e}$, but this requires perhaps further experimental confirmation.

According to the well-known isotope effect of confinement, the heat transport is observed experimentally to be higher in hydrogen than in deuterium, [49, 50, 51]. It should be underlined that this difference is opposite to that expected from gyro-Bohm transport, as $\rho^{*}$ is smaller in hydrogen than in deuterium at fixed temperature. The higher transport in hydrogen observed experimentally is a possible hypothesis to explain the higher values of $P_{L-H}$ and $Q_{i, e d g e}^{L-H}$ in this gas because the required value of $E_{r}$ and therefore $\nabla p_{i} / n_{i}$ at the plasma edge can be expected to require a higher $Q_{i, e d g e}$. This can be investigated with our experimental data.

In figure 10 we plot $T_{i, e d g e}$ at the $\mathrm{L}-\mathrm{H}$ transition for hydrogen and deuterium. The hydrogen values are only marginally higher than in deuterium and the density dependence is weak in both gases. It should be underlined that the deuterium data are in agreement with the previous results obtained in ASDEX Upgrade, [22]. Similar edge temperatures at the L-H transition in hydrogen and deuterium have also been observed in DIII-D, [15].

The corresponding values of $\nabla T_{i}$ plotted in figure 11 indicate that, at the L-H transition, the gradient is the same in deuterium and hydrogen, within the experimental uncertainties, and independent of the density for both ion species. Therefore, $\nabla p_{i} /\left(e \cdot n_{i}\right)$ is similar in 


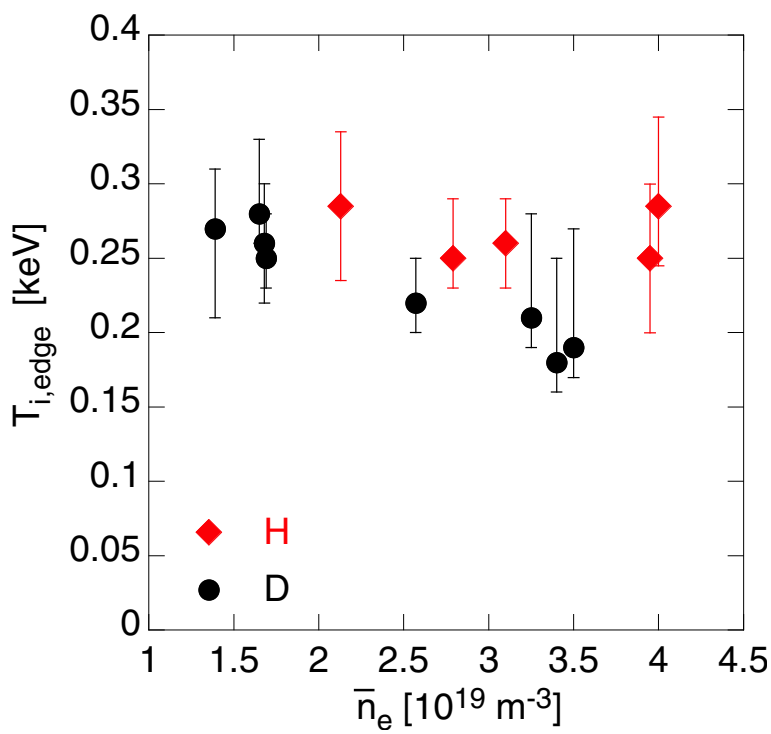

Figure 10. $T_{i, e d g e}$ at the $L-H$ transition versus line-averaged density for hydrogen and deuterium.

hydrogen and deuterium, suggesting that the $E_{r}$ is also similar. This is in agreement with an independent study carried out at ASDEX Upgrade in different dedicated discharges in which the $E_{r}$ profile has been obtained from the CXRS data applying the ion force balance to the measured impurity and which indeed indicates that the $E_{r}$ well at the L-H transition is very similar in hydrogen and deuterium, [38].

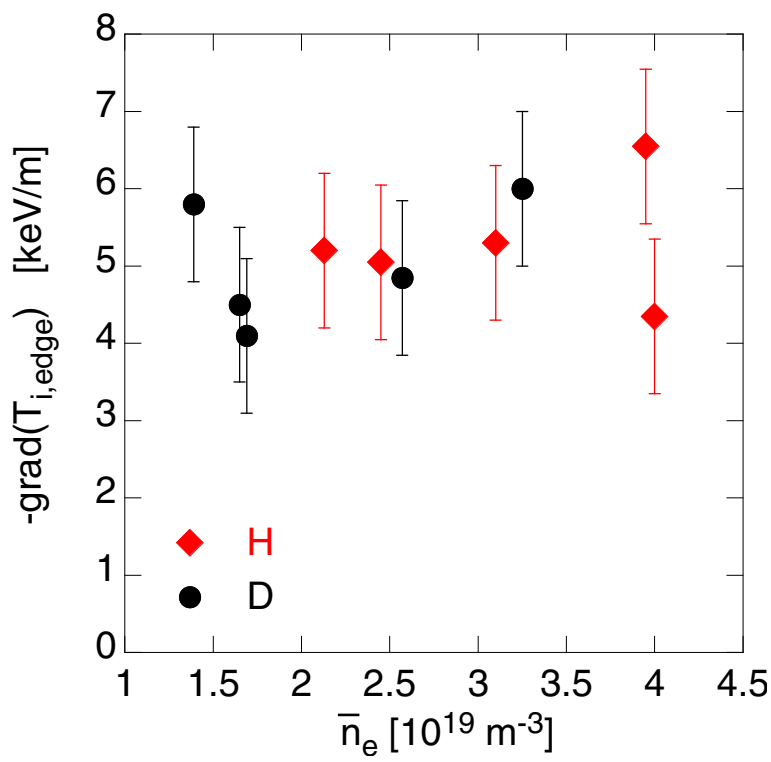

Figure 11. $-\nabla T_{i, e d g e}$ at the $L-H$ transition versus line-averaged density for hydrogen and deuterium. 
These experimental data at the L-H transition at different densities can be summarised by using $Q_{i, e d g e} / n_{i, e d g e}$ to take the density range into account. This quantity is plotted versus $-\nabla T_{i}$ for hydrogen and deuterium in figure 12. As could be anticipated from figure 8 , $Q_{i, e d g e}^{L-H} / n_{i, e d g e}$ at the $\mathrm{L}-\mathrm{H}$ transition is clearly higher in hydrogen compared to deuterium while $\nabla T_{i, e d g e}^{L-H}$ almost does not change. As $T_{i, e d g e}^{L-H}$ varies little, the values of the ion diffusivity, which can be deduced from these data sets, can be compared. These are indicated in figure 12 and yield: $\chi_{i, e d g e}^{H}=2.2 \pm 0.5 \mathrm{~m}^{2} / \mathrm{s}$ and $\chi_{i, e d g e}^{D}=1.05 \pm 0.35 \mathrm{~m}^{2} / \mathrm{s}$ in hydrogen and deuterium respectively.

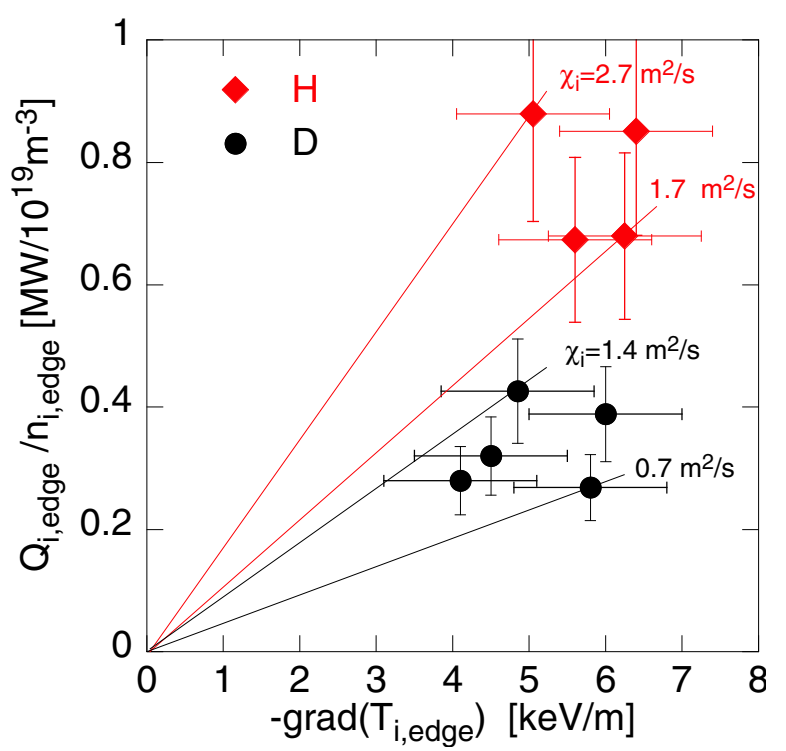

Figure 12. $Q_{i, e d g e} / n_{i, e d g e}$ versus $-\nabla T_{i, \text { edge }}$ at $\rho_{\text {pol }} \approx 0.98$ for hydrogen and deuterium at the L-H transition. The corresponding values for the ion heat diffusion coefficient are indicated in the plot.

Therefore, despite the large experimental uncertainties, this result strongly suggests that, at the plasma edge, the ion heat transport in hydrogen is higher than in deuterium, which leads to a higher L-H power threshold in hydrogen to stabilise turbulence through $\nabla E_{r}$. The turbulence measurements reported in [52] for the L-mode isotope and $\rho^{*}$ transport study carried out in ASDEX Upgrade indicate that the radial correlation length of the turbulence measured by reflectometry in hydrogen and deuterium is very similar in the radial region at the plasma edge, $0.95<\rho_{\text {pol }}<1.0$. Thus, the assumption that a similar $E_{r}$ well is required for turbulence stabilisation is plausible. As reported in the same reference, in the scrapeoff layer the correlation length of the turbulence measured by the Li-beam beam emission 
spectroscopy, in the same discharges, is about $25 \%$ larger in D than in H. Further studies along this line would be required to document the turbulence behaviour and a possible relation with the $\nabla E_{r}$ stabilisation in the two gases at the L-H transition.

\section{Summary and conclusion}

Our previous investigations on the key role played by the edge ion heat flux in the $\mathrm{L}-\mathrm{H}$ transition, carried out in deuterium plasmas, [11], have been extended in hydrogen. We analysed the well of the edge radial electric field in L-mode up to the L-H transition and obtained indications that it is very similar in hydrogen and deuterium at the L-H transition, see [38] for a comprehensive analysis of the $E_{r}$ well and its comparison to the neoclassical prediction, as well as the discussion of the possible role of turbulence-induced flows in the L-H transition mechanism at ASDEX Upgrade.

At the L-H transition, the edge ion heat flux in hydrogen is about two times higher than in deuterium which can be clearly attributed to the well-known isotope effect: the ion heat transport is higher in hydrogen. In both hydrogen and deuterium the non-monotonic density dependence of the power threshold, with its minimum at $n_{e, \min }$, whereby $P_{L-H}$ increases towards low densities, is attributed to the decoupling of the electron and ion heat channels: more power is required to reach the value of the edge ion heat flux which establishes the $E_{r}$ well necessary to stabilise the turbulence. Based on the interpretation of $n_{e, \min }$ presented in [11], the somewhat higher value of $n_{e, \min }$ in hydrogen is attributed to the lower confinement time in this gas, according to the well-known isotope effect of confinement.

Finally a word of caution: Our results have been obtained at fixed magnetic field and for a given magnetic configuration, such that the dependence of $Q_{i, e d g e}^{L-H}$ on density presented here might vary if the conditions are different. Further, as the profiles of $T_{i}, n_{i}$ and $E_{r}$ at the very edge of the plasma might be influenced by the scrape-off layer and divertor conditions, the edge ion heat flux is an important player in determining $E_{r}$, but probably not the only one. Thus, the $\mathrm{E} \times \mathrm{B}$ shear might be not solely determined by the neoclassical effects but also by the scrape-off layer. In particular, it should be underlined that the contribution of $T_{i} \nabla n_{i} / n_{i}$ to $\nabla p_{i} /\left(e \cdot n_{i}\right)$ is about two thirds, such that small variations of $\nabla n_{i} / n_{i}$ can play a significant role. The latter can be influenced by the plasma wall interaction and divertor conditions. Variations of $\nabla n_{i} / n_{i}$ are indeed proposed as a possible explanation in ASDEX Upgrade for the reduction of the power threshold observed in the metallic wall compared to the carbon wall, [53]. This 
effect might also be one of the reasons for the variations of the low density branch and $n_{e, \text { min }}$ observed in JET, [9].

\section{Acknowledgement}

It is a pleasure to acknowledge the excellent support of the ASDEX Upgrade technical staff. This work has been carried out within the framework of the EUROfusion Consortium and has received funding from the Euratom research and training programme 2014-2018 under grant agreement No 633053. The views and opinions expressed herein do not necessarily reflect those of the European Commission.

\section{References}

[1] F. Ryter et al., Nuclear Fusion, 36 (1996) 1217.

[2] S. J. Fielding et al., Plasma Phys. Controlled Fusion 40 (1998) 731.

[3] T. Fukuda et al., Plasma Phys. Controlled Fusion 42 (2000) A289.

[4] Y. Andrew et al., Plasma Physics and Controlled Fusion 48 (2006) 479.

[5] F. Ryter et al., Nuclear Fusion 49 (2009) 062003.

[6] P. Gohil et al., Nuclear Fusion 49 (2009) 115004.

[7] Y. Ma et al., Nuclear Fusion 52 (2012) 023010.

[8] F. Ryter et al., Nuclear Fusion 53 (2013) 113003.

[9] C. Maggi et al., Nuclear Fusion 54 (2014) 023007.

[10] F. Ryter et al., Plasma Physics and Controlled Fusion 36 (1994) 99.

[11] F. Ryter et al., Nuclear Fusion 54 (2014) 083003.

[12] The ASDEX Team, Nucl. Fusion 29 (1989) 1959.

[13] K. Lackner et al., Plasma Physics and Controlled Fusion 36 (1994) B79.

[14] E. Righi et al., Nucl. Fusion 39 (1999) 309.

[15] P. Gohil et al., Nuclear Fusion 50 (2010) 064011.

[16] D. C. McDonald et al., Plasma Physics and Controlled Fusion 46 (2004) 519.

[17] P. Gohil et al., Nuclear Fusion 51 (2011) 103020.

[18] H. Biglari et al., Phys. Fluids B 2 (1990) 1.

[19] R. J. Groebner et al., Phys. Rev. Lett. 64 (1990) 3015.

[20] K. H. Burrell, Phys. Plasmas 4 (1997) 1499.

[21] F. Wagner, Plasma Physics and Controlled Fusion 49 (2007) B1.

[22] P. Sauter et al., Nuclear Fusion 52 (2012) 012001.

[23] E. Viezzer et al., Nuclear Fusion 53 (2013) 053005.

[24] F. L. Hinton et al., Rev. Mod. Phys. 48 (1976) 239.

[25] R. M. McDermott et al., Physics of Plasmas 16 (2009) 056103. 
[26] U. Stroth et al., Plasma Physics and Controlled Fusion 53 (2011) 024006.

[27] R. A. Moyer et al., Phys. Rev. Lett. 87 (2001) 135001.

[28] R. J. Colchin et al., Phys. Rev. Lett. 88 (2002) 255002.

[29] H. Punzmann et al., Phys. Rev. Lett. 93 (2004) 125003.

[30] G. D. Conway et al., Phys. Rev. Lett. 106 (2011) 065001.

[31] T. Estrada et al., Phys. Rev. Lett. 107 (2011) 245004.

[32] L. Schmitz et al., Phys. Rev. Lett. 108 (2012) 155002.

[33] G. Tynan et al., Nuclear Fusion 53 (2013) 073053.

[34] E. J. Kim et al., Phys. Rev. Lett. 90 (2003) 185006.

[35] S. K. Rathgeber et al., Plasma Physics and Controlled Fusion 55 (2013) 025004.

[36] R. Fischer et al., Fusion Science and Technology 58 (2010) 675.

[37] E. Viezzer et al., Review of Scientific Instruments 83 (2012) 103501.

[38] M. Cavedon et al., in 42nd EPS Conference on Plasma physics, paper P1.130, 2015, in preparation for Phys. Rev. Letters.

[39] R. M. McDermott et al., Plasma Physics and Controlled Fusion 53 (2011) 124013.

[40] G. D. Conway, Plasma Physics and Controlled Fusion 50 (2008) 124026.

[41] A. Pankin et al., Computer Phys. Comm. 159 (2004) 157.

[42] F. Leuterer et al., Nuclear Fusion 43 (2003) 1329.

[43] A. Mlynek et al., Review of Scientific Instruments 81 (2010) 033507.

[44] C. Angioni et al., Nuclear Fusion 44 (2004) 827.

[45] A. Mlynek et al., Nuclear Fusion 52 (2012) 114012.

[46] A. G. Peeters, Physics of Plasmas 7 (2000) 268.

[47] E. Viezzer et al., Nuclear Fusion 54 (2014) 012003.

[48] E. Delabie, Private communication .

[49] M. Bessenrodt-Weberpals et al., Nucl. Fusion 33 (1993) 1205.

[50] F. Wagner et al., Plasma Phys. Controlled Fusion 35 (1993) 1321.

[51] U. Stroth, Plasma Phys. Controlled Fusion 40 (1998) 9.

[52] P. Hennequin et al., in 42nd EPS Conference on Plasma physics, paper I1.102, 2015, submitted to Plasma Phys. Control. Fus.

[53] L. Shao et al., in 42nd EPS Conference on Plasma physics, paper P1.113, 2015, in preparation for Plasma Phys. Control. Fus. 